\title{
Improved Method for Preparation of Methoxy-Modified Kaolinite
}

\author{
Ding Wang, ${ }^{a}$ Qinfu Liu, ${ }^{* a}{ }^{a}$ Dandan Hou, ${ }^{a}$ Shuai Zhang, ${ }^{a}$ Peng Guo ${ }^{b}$ and Hongfei Cheng ${ }^{a}$ \\ ${ }^{a}$ School of Geoscience and Surveying Engineering, China University of Mining \& Technology, \\ 100083 Beijing, China \\ ${ }^{b}$ No. 1 Geological Exploration Institute of Henan Province, 450001 Zhengzhou, China
}

\begin{abstract}
A hydrated kaolinite-methanol intercalation compound (Kaol-MeOH (hydrated)) was synthesized by utilizing kaolinite/dimethyl sulfoxide intercalation compound (Kaol-DMSO) as an intermediary, and further displacement intercalation with butyl trimethylammonium chloride (BTAC) was also carried out. The obtained samples were characterized by X-ray diffraction (XRD) and Fourier transform infrared spectroscopy (FTIR). Results showed that the $d_{001}$ value of the Kaol-MeOH (hydrated) could be arbitrary values between 0.86 and $1.08 \mathrm{~nm}$, which could be caused by the hydrogen-bonded $\mathrm{MeOH}$ or water molecules between the interlayer spaces of methoxy-modified Kaol. Furthermore, this part of interlayer molecules could be removed by heating and have no obvious effect on the subsequent intercalation reaction. In addition, the time required for Kaol- $\mathrm{MeOH}$ (hydrated) preparation was drastically shortened to 3 days, which greatly reduces the time-consuming for the preparation of subsequent compounds.
\end{abstract}

Keywords: kaolinite, methanol, hydrated, intercalation

\section{Introduction}

Kaolinite (Kaol) is one of the most well-known clay minerals. It is a typical 1:1 clay mineral, which is composed of tetrahedral silica $(\mathrm{Si}-\mathrm{O})$ sheet and an octahedral alumina $(\mathrm{Al}-\mathrm{OH})$ sheet along the c-axis. The tetrahedral sheets share a layer of oxygen atoms with the octahedral sheets and these two sheets bond closely together by covalent bonds. Thus, the energetic barrier of the hydrogen bonds must be overcome before the intercalation of Kaol, and only a limited number of polar organic molecules can be directly intercalated, such as dimethyl sulfoxide (DMSO), urea (U), dimethyl formamide (DMF), $N$-methylformamide (NMF), etc. Theng ${ }^{1}$ reported that displacement reactions could be used as an effective way to extend the intercalation of Kaol with a wide range of guest substances. Subsequently, many different types of organic species were introduced into Kaol interlayers by this method, such as $\gamma$-aminopropyl triethoxysilane, ${ }^{2}$ ammonium acetate,${ }^{3}$ lactam, ${ }^{4}$ acrylamide, ${ }^{5}$ ethylene glycol, ${ }^{6}$ amino alcohols ${ }^{7}$ and $\mathrm{MeOH}$.

Methoxy-modified Kaol as a highly versatile intermediate/intermediary was first reported by Raythatha and Lipsicas, ${ }^{8}$ in which $\mathrm{MeOH}$ was utilized to wash

*e-mail: lqf@ cumtb.edu.cn
Kaol/DMSO intercalation compound for synthesizing hydrated Kaol. Then, methoxy-functionalized kaolinite was used as an intermediate to prepare a hydrated phase of Kaol by Tunney and Detellier. ${ }^{9}$ Subsequently, Komori et al. ${ }^{10-12}$ discovered an easy way to prepare $\mathrm{MeOH}$-intercalated kaolinite at room temperature. After that, a number of organic substances, such as alkylamine, ${ }^{13-15}$ quaternary ammonium salt ${ }^{16,17}$ and stearic acid, ${ }^{18}$ were introduced into the layers of Kaol by using a methoxy-modified Kaol as a precursor. DMSO, NMF, and U intercalated Kaol could be used to graft methoxy groups via reaction with $\mathrm{MeOH}$. However, Matusik et al. ${ }^{19-21}$ reported that dry kaolinite intercalated with DMSO used as an intermediate was the most favorable condition for the synthesis of Kaol-MeOH.

Details on preparation and characterization of methoxymodified Kaol have been discussed in above literatures, while the preparation process of this highly versatile intermediate usually takes at least seven days. Furthermore, quaternary alkylammonium ions, as an important modifier, are the most used organic compounds to prepare organoclays and widely used in industries application. Herein we explored the feasibility of preparing Kaol$\mathrm{MeOH}$ in a relatively short time and the final products were employed to intercalation with butyl trimethylammonium chloride (BTAC). X-ray diffraction (XRD) combined with 
Fourier transform infrared spectroscopy (FTIR) was used to characterize the methoxy-modified Kaol produced by this method. This method is expected to be useful for producing nanocomposites in a relatively short time.

\section{Experimental}

\section{Materials}

A Kaol specimen consisting mainly of mineral kaolinite (95 mass\%), obtained from Hebei province in China, was used as received without further purification. The chemical composition of the sample was detailed in our previous studies. ${ }^{22,23}$ The sample is well crystallized with a Hinckley index of ca. 1.31. DMSO, $\mathrm{MeOH}$ (purity $\geq 99.5 \%$ ), ethanol (purity $\geq 99.5 \%$ ), and BTAC were purchased from Adamas Reagent Co., Ltd (China). All reagents were of analytical grade purity and used as received.

\section{Synthesis of intercalation compounds}

Kaol-DMSO intercalation compound was prepared by adding $10 \mathrm{~g}$ Kaol into a mixture of $180 \mathrm{~mL}$ DMSO and $20 \mathrm{~mL}$ deionized water. The mixture was stirred at $60^{\circ} \mathrm{C}$ for $12 \mathrm{~h}$, and then centrifuged. For further use, the obtained product was dried in an oven under $60{ }^{\circ} \mathrm{C}$ for $12 \mathrm{~h}$.

Methoxy-modified Kaol was prepared by adding $2 \mathrm{~g}$ of Kaol-DMSO into $100 \mathrm{~mL}$ of $\mathrm{MeOH}$ (solid-to-liquid ratio was 1:50), which was much larger than the conventional method. ${ }^{10-12,24,25}$ The dispersion was stirred for 3 days and replaced by fresh $\mathrm{MeOH}$ every day. Then, the solid in the mixture was separated via centrifugation ( $3 \mathrm{krpm}, 10 \mathrm{~min}$ ), and dried in an oven at $60{ }^{\circ} \mathrm{C}$ for $12 \mathrm{~h}$. The solid samples were denoted as $\mathrm{Kaol}-\mathrm{MeOH}$ (hydrated). As a reference, a conventional method was also utilized to prepare methoxymodified Kaol. The sample was denoted as Kaol-MeOH.

In order to investigate the structure of Kaol-MeOH (hydrated), further experiments were conducted. Firstly, Kaol-MeOH (hydrated) was placed in an oven and dried at $80^{\circ} \mathrm{C}$ and examined by XRD every two hours. On the other hand, a gram of Kaol-MeOH (hydrated) was dispersed in a $10 \mathrm{~mL}$ solution containing $1 \mathrm{~mol} \mathrm{~L}^{-1} \mathrm{BTAC}$ in $\mathrm{MeOH}$ and stirred with a magnetic stirrer for $24 \mathrm{~h}$. The solid in the mixture was then separated by centrifugation $(3 \mathrm{krpm}$, $10 \mathrm{~min}$ ), extensively washed 3 times with fresh ethanol to remove the BTAC in excess, and then dried in an oven at $60{ }^{\circ} \mathrm{C}$ for $4 \mathrm{~h}$. The product was denoted as Kaol-BTAC.

\section{Characterization}

XRD patterns were recorded on a Rigaku D/MAX 2500
PC instrument, which was equipped with $\mathrm{Ni}$-filtered and $\mathrm{Cu} \mathrm{K} \alpha$ radiation. The instrument was operated at $45 \mathrm{kV}$ and $100 \mathrm{~mA}$ and the sample scanned in the range of $2.5-15^{\circ}$ with a speed of $2^{\circ} \mathrm{min}^{-1}$. FTIR spectra were collected on the Thermo Fisher Nicolet 6700 spectrometer by a $\mathrm{KBr}$ disk (ca. $2 \%$ by mass in $\mathrm{KBr}$ ) from 600 to $4,000 \mathrm{~cm}^{-1}$ at $4 \mathrm{~cm}^{-1}$ resolution. 512 spectra were recorded, which were co-added to improve the signal-to-noise ratio.

\section{Results and Discussion}

\section{XRD analysis}

The XRD patterns of Kaol, Kaol-DMSO, Kaol-MeOH (wet), Kaol-MeOH (dry) and $\mathrm{Kaol}-\mathrm{MeOH}$ (hydrated) are shown in Figure 1. The Kaol and Kaol-DMSO showed the typical basal spacings of 0.72 and $1.13 \mathrm{~nm}$, respectively. ${ }^{26,27}$ The basal peak of the Kaol-MeOH (wet), the $\mathrm{MeOH}$ intercalated Kaol obtained under wet condition, was observed at $1.08 \mathrm{~nm}$, which is in good agreement with published data. This is a typical interlayer structure of methoxy-modified Kaol under wet state, in which a monolayer $\mathrm{MeOH}$ molecules was presented between the siloxane surface and the methoxy-modified inner surface. ${ }^{9}$ Methoxy groups grafted on the aluminol surface of Kaol via $\mathrm{Al}-\mathrm{O}-\mathrm{C}$ bond resulted in the methoxy-modified inner surface, accompanied by the loss of hydrogen of $\mathrm{MeOH}$. Furthermore, a small number of water molecules may be generated by combing the loss hydrogen of $\mathrm{MeOH}$ and the hydroxyl groups of Kaol.

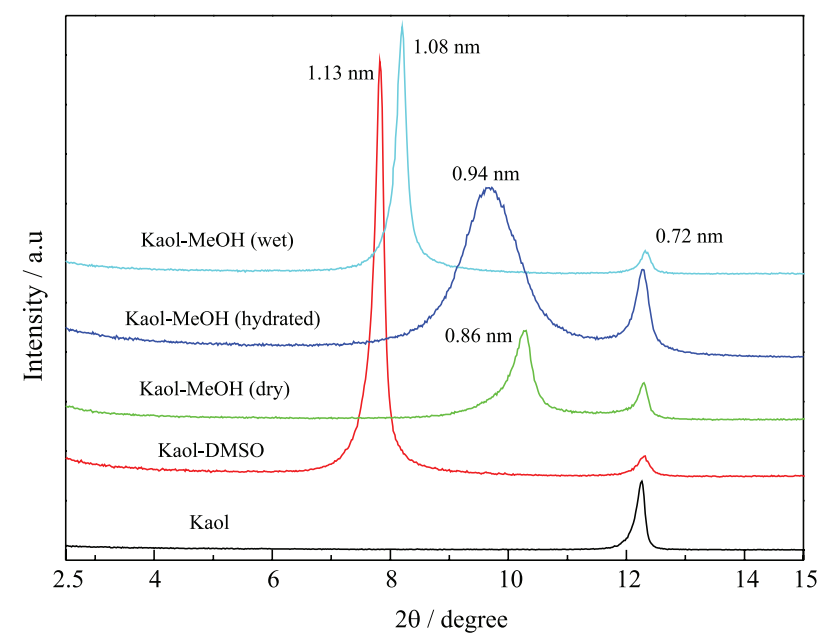

Figure 1. XRD patterns of Kaol, Kaol-DMSO, Kaol-MeOH (wet), $\mathrm{Kaol}-\mathrm{MeOH}$ (dry) and Kaol-MeOH (hydrated).

Compared with Kaol-MeOH (wet), after the drying process, the basal reflection of Kaol-MeOH (dry) was reduced to $0.86 \mathrm{~nm}$, which was attributed to the escape 
of $\mathrm{MeOH}$ molecules from the interlayer space. According to previous literature, ${ }^{9,11}$ the $d_{001}$ value of non-hydrated $\mathrm{Kaol}-\mathrm{MeOH}$ was $0.82 \mathrm{~nm}$, which was smaller than that of Kaol-MeOH (dry). Therefore, the Kaol-MeOH (dry) was partially hydrated, and a number of hydrogen-bonded $\mathrm{MeOH}$ or water molecules were likely presented in the Kaol interlayer space. The hydrogen bonds could be formed by the $\mathrm{MeOH}$ molecules or water molecules with the un-grafted hydroxyl groups. The XRD pattern of Kaol-MeOH showed the basal spacing of $1.08 \mathrm{~nm}$ under wet state and $0.86 \mathrm{~nm}$ after drying. On the other hand, a new reflection at $0.94 \mathrm{~nm}$ (arbitrary values between 0.86 and $1.08 \mathrm{~nm}$, actually) was observed in the pattern of $\mathrm{Kaol}-\mathrm{MeOH}$ (hydrated), indicating that a larger number of $\mathrm{MeOH}$ or water molecules may presented in the interlayer space of Kaol than that of Kaol-MeOH (dry).

The obtained Kaol-MeOH (hydrated) was heated in an oven under $80^{\circ} \mathrm{C}$, and the XRD patterns of the sample were recorded every two hours. The XRD patterns of Kaol-MeOH (hydrated) are displayed in Figure 2 (left), and the relation between the intercalation ratio and the time of drying is plotted in Figure 2 (right). A nearly linear relationship was recognized (Figure 2 (left)), and the reflections shifted to higher degree with the increased time of drying. The basal peak of final products reached to $0.87 \mathrm{~nm}$ after $6 \mathrm{~h}$. During the drying process, the intensity of the reflection peak became weaker, accompanied by the decreasing intercalation ratio and the wider full width at half-height. These results also demonstrated that the methoxy-modified Kaol produced by this method was hydrated, and part of hydrogen-bonded $\mathrm{MeOH}$ or water molecules was presented in the interlayers of Kaol. Furthermore, this part of $\mathrm{MeOH}$ or water molecules could be removed by heating.

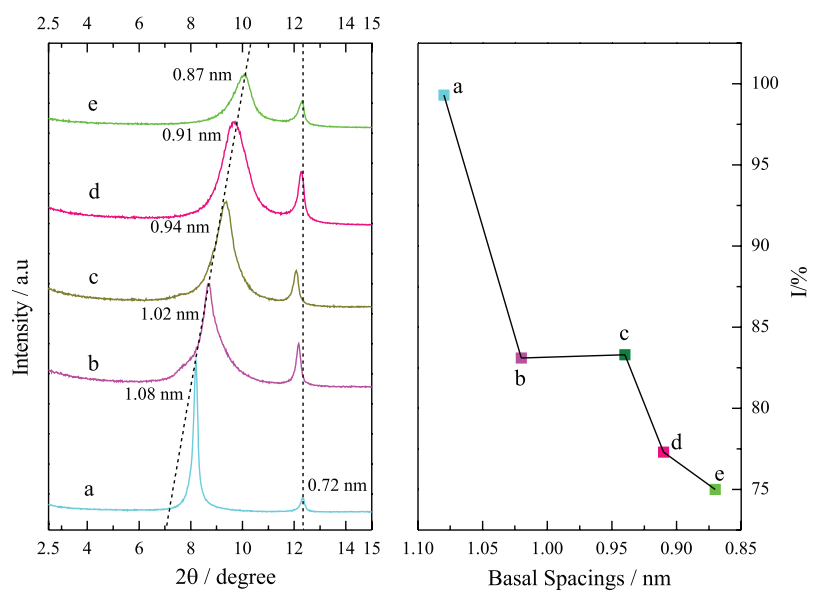

Figure 2. (Left) XRD patterns of (a) Kaol-MeOH (wet), (b) Kaol-MeOH (hydrated) at room temperature and after drying for (c) $2 \mathrm{~h}$, (d) $4 \mathrm{~h}$, (e) $6 \mathrm{~h}$; (right) its corresponding variation in intercalation ratio with time of drying.
The solid-to-liquid ratio of Kaol-DMSO to $\mathrm{MeOH}$ of 1:100 and 1:200 were also used to investigate the effect of $\mathrm{MeOH}$ concentration on the intercalation. As shown in Figure 3, the variation in the basal spacings was nearly consistent with that of 1:50 after 3 days, indicating that the solid-to-liquid of Kaol-DMSO to $\mathrm{MeOH}$ of 1:50 was sufficient to prepare the methoxy-modified Kaol.
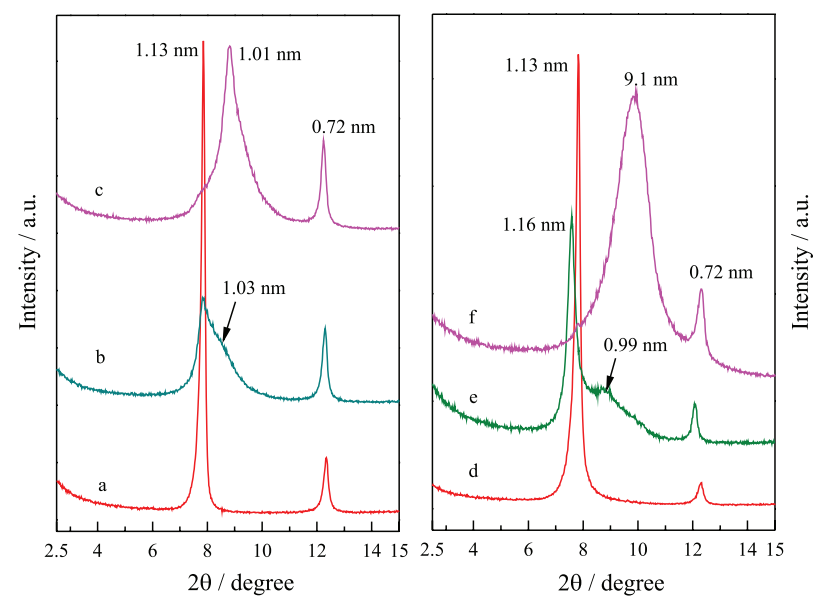

Figure 3. XRD patterns of $\mathrm{Kaol}-\mathrm{MeOH}$ (hydrated) prepared at the solid-to-liquid ratio of Kaol-DMSO to $\mathrm{MeOH}$ of 1:100 (left) and 1:200 (right): (a) and (d) conducted for 1 day; (b) and (e) conducted for 2 days; (c) and (f) conducted for 3 days.

\section{FTIR spectra}

Figure 4 shows the $\mathrm{KBr}$ spectra of Kaol, Kaol-DMSO, $\mathrm{Kaol}-\mathrm{MeOH}$ (dry) and Kaol-MeOH (hydrated). Kaol exhibited four absorption bands in the $\mathrm{OH}$ stretching region. The absorption near $3620 \mathrm{~cm}^{-1}$ is ascribed to the inner hydroxyl groups, which was lying between the tetrahedral and octahedral sheets. Residing at the octahedral surface of Kaol, the other three $\mathrm{OH}$ groups form hydrogen bonds with the oxygens of the $\mathrm{Si}-\mathrm{O}-\mathrm{Si}$ bonds on the lower surface of the next layer. The strong absorption at $3686 \mathrm{~cm}^{-1}$ was ascribed to the in-phase symmetric stretching vibration, and two weak bands at 3675 and $3652 \mathrm{~cm}^{-1}$ are related to out-of-plane stretching vibrations. The band at $912 \mathrm{~cm}^{-1}$ is assigned to the bending vibrations of the inner hydroxyls, which was not affected by intercalation of guest molecules. ${ }^{28,29}$

Upon intercalation of DMSO, bands observed at 1317 and $1427 \mathrm{~cm}^{-1}$ are assigned to the vibrational peaks of methyl groups in DMSO molecules. Two weak bands at 3455 and $1631 \mathrm{~cm}^{-1}$ are caused by the $\mathrm{OH}$ stretching of absorbed water. Bands at ca. 2920 and ca. $2850 \mathrm{~cm}^{-1}$ corresponded to $\mathrm{CH}_{3}$ stretching vibrations of residual DMSO. After methoxy-grafting, the inner surface hydroxyl bands at 3686 and $3652 \mathrm{~cm}^{-1}$ decreased significantly, while the band at $3620 \mathrm{~cm}^{-1}$ did not. Compared with Kaol- 


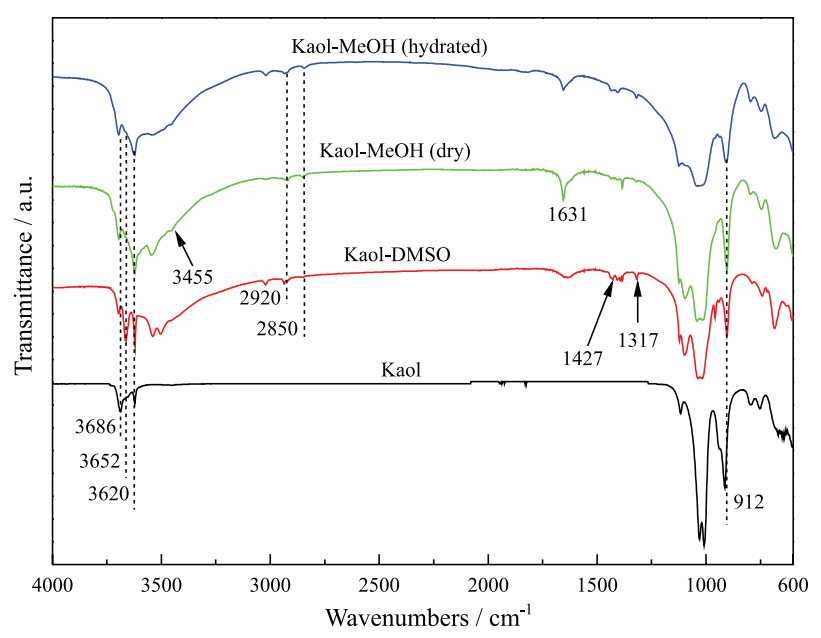

Figure 4. FTIR spectra of Kaol, Kaol-DMSO, Kaol-MeOH (dry) and Kaol-MeOH (hydrated).

$\mathrm{MeOH}$ (dry), a broader and weaker band at $3455 \mathrm{~cm}^{-1}$ was observed in the spectra of Kaol-MeOH (hydrated), which means much more water molecules were adsorbed on the Kaol-MeOH (hydrated).

\section{Preparation of Kaol-BTAC intercalation compound}

Kaol-MeOH (hydrated) was further used to intercalate with BTAC and the XRD patterns of Kaol-MeOH (hydrated) and Kaol-BTAC are shown in Figure 5. The Kaol-BTAC intercalation compound exhibits the first basal peak with a $d_{001}$ value of $1.39 \mathrm{~nm}$, which was in good agreement with our previous report, ${ }^{30}$ indicating that the hydrogen-bonded

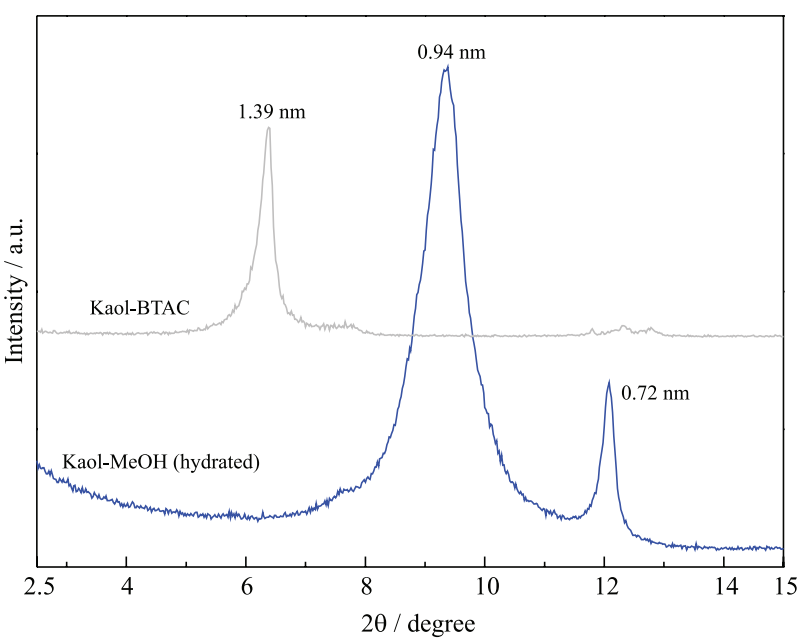

Figure 5. XRD patterns of Kaol-MeOH (hydrated) and Kaol-BTAC.

$\mathrm{MeOH}$ or water molecules has no obvious effect on the intercalation reaction.

Some efforts have been done to study the arrangement models of alkyl chains between the Kaol interlayer spaces. ${ }^{16,17,30-35}$ Compared with Kaol-MeOH (dry) $(0.86 \mathrm{~nm})$, the increment of basal spacing of Kaol-BTAC was ca. $0.51 \mathrm{~nm}$, which was approximately in accordance with the van der Waals radii of the hydrocarbon chain. ${ }^{36}$ When $\mathrm{BTAC}^{+}$is lying flat, the heights of the head group and that of alkyl chain are approximately 0.51 and $0.41 \mathrm{~nm}$, respectively. Therefore, according to our previous report, ${ }^{30}$ it is reasonable to suggest that the surfactant in the interlayer space of Kaol adopt a lateral-monolayer arrangement, as shown in Figure 6.

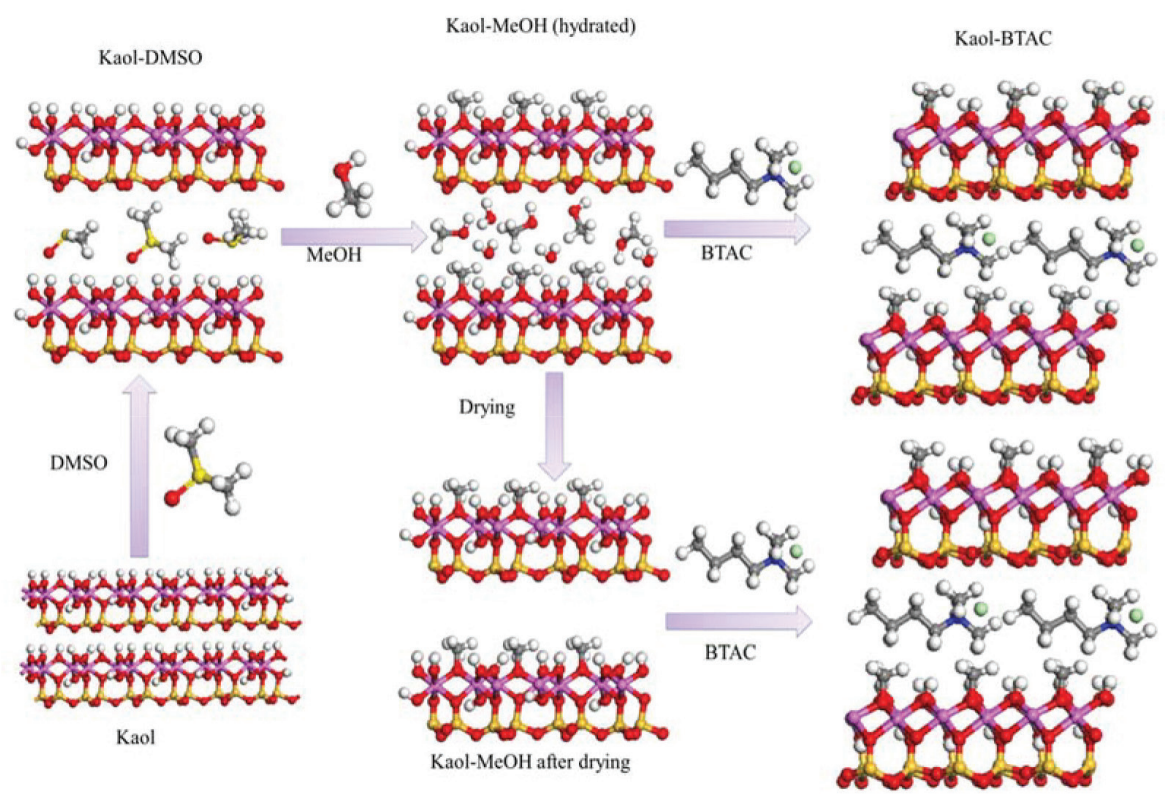

Kaol-BTAC

Figure 6. Schematic representation of the transformation from Kaol to Kaol-BTAC. 


\section{Conclusions}

The method for preparation of methoxy-modified Kaol was improved, in which the time required for preparation was shortened by more than fifty percent. The obtained products were hydrated and the $d_{001}$ value could be any value between 1.08 and $0.86 \mathrm{~nm}$, which was resulted from a number of hydrogen-bonded $\mathrm{MeOH}$ or water molecules presented in the interlayer space of Kaol. Nevertheless, the properties of the products were not affected by the interlayer $\mathrm{MeOH}$ or water molecules and the products could be used for the subsequent studies.

\section{Supplementary Information}

Supplementary information is available free of charge at http://jbcs.sbq.org.br as PDF file.

\section{Acknowledgments}

The authors gratefully acknowledge the financial support provided by the National Natural Science Foundation of China (51034006), Beijing Natural Science Foundation (8164062), Beijing Nova Program (xx2015B081) and Beijing talent plan (2014000020124G164).

\section{References}

1. Theng, B. K. G.; The Chemistry of Clay-Organic Reactions; John Wiley and Sons: New York, USA, 1974.

2. Zhang, S.; Liu, Q. F.; Cheng, H. F.; Zhang, Y. D.; Li, X. G.; Frost, R. L.; Appl. Clay Sci. 2015, 114, 484.

3. Sugahara, Y.; Satokawa, S.; Kuroda, K.; Kato, C.; Clays Clay Miner. 1988, 36, 343.

4. Sugahara, Y.; Kitano, S.; Satokawa, S.; Kuroda, K.; Kato, C.; Bull. Chem. Soc. Jpn. 1986, 59, 2607.

5. Sugahara, Y.; Satokawa, S.; Kuroda, K.; Kato, C.; Clays Clay Miner. 1990, 38, 137.

6. Tunney, J. J.; Detellier, C.; Chem. Mater. 1993, 5, 747.

7. Tunney, J. J.; Detellier, C.; Can. J. Chem. 1997, 75, 1766.

8. Raythatha, R.; Lipsicas, M.; Clays Clay Miner. 1985, 33, 333.

9. Tunney, J. J.; Detellier, C.; J. Mater. Chem. 1996, 6, 1679.

10. Komori, Y.; Sugahara, Y.; Kuroda, K.; Appl. Clay Sci. 1999, 15 , 241.

11. Komori, Y.; Enoto, H.; Takenawa, R.; Hayashi, S.; Sugahara, Y.; Kuroda, K.; Langmuir 2000, 16, 5506.

12. Komori, Y.; Sugahara, Y.; Kuroda, K.; J. Mater. Res. 1998, 13, 930.
13. Gardolinski, J.; Lagaly, G.; Clay Miner. 2005, 40, 547.

14. Gardolinski, J.; Lagaly, G.; Clay Miner. 2005, 40, 537.

15. Liu, Q. F.; Li, X. G.; Guo, P.; Cheng, H. F.; Ji, L. B.; Zhang, S.; J. Chin. Ceram. Soc. 2014, 42, 1064.

16. Kuroda, Y.; Ito, K.; Itabashi, K.; Kuroda, K.; Langmuir 2011, 27, 2028.

17. Yuan, P.; Tan, D. Y.; Annabi-Bergaya, F.; Yan, W. C.; Liu, D.; Liu, Z. W.; Appl. Clay Sci. 2013, 83-84, 68.

18. Liu, Q. F.; Zuo, X. C.; Zhang, S. L.; Zhang, S.; Ji, J. C.; Chin. J. Inorg. Chem. 2015, 31, 7.

19. Matusik, J.; Gaweł, A.; Bielańska, E.; Osuch, W.; Bahranowski, K.; Clays Clay Miner. 2009, 57, 452.

20. Matusik, J.; Gaweł, A.; Bahranowski, K.; Appl. Clay Sci. 2012, $56,63$.

21. Matusik, J.; Scholtzová, E.; Tunega, D.; Clays Clay Miner. 2012, 60, 227.

22. Cheng, H. F.; Xu, P. J.; Wang, D.; Frost, L. R.; J. Therm. Anal. Calorim. 2016, 126, 421.

23. Liu, Q. F.; Zhang, S.; Cheng, H. F.; Wang, D.; Li, X. G.; Hou, X. J.; Frost, L. R.; J. Therm. Anal. Calorim. 2014, 117, 189.

24. Wang, S.; Zuo, X. C.; Cheng, H. F.; Yang, Y. J.; Liu, Q. F.; J. Braz. Chem. Soc. 2016, 27, 1311.

25. Makó, É.; Kovács, A.; Ható, Z.; Kristóf, T.; Appl. Surf. Sci. 2015, 357, 626.

26. Cheng, H. F.; Liu, Q. F.; Yang, J.; Ma, S. J.; Frost, R. L.; Thermochim. Acta 2012, 545, 1.

27. Zhang, S.; Liu, Q. F.; Cheng, H. F.; Li, X. G.; Zeng, F. G.; Frost, R. L.; J. Colloid Interface Sci. 2014, 430, 345.

28. Caglar, B.; Çırak, Ç.; Tabak, A.; Afsin, B.; Eren, E.; J. Mol. Struct. 2013, 1032, 12.

29. Elbokl, T. A.; Detellier, C.; J. Colloid Interface Sci. 2008, 323, 338.

30. Liu, Q. F.; Wang, D.; Guo, P.; Zhang, S.; Cheng, H. F.; Li, X. G.; Zhang, S. L.; J. Chin. Ceram. Soc. 2015, 43, 222.

31. Venkataraman, N.; Vasudevan, S.; J. Phys. Chem. B 2011, 105, 1805.

32. Lagaly, G.; Clay Miner. 1981, 16, 1.

33. Cheng, H. F.; Xu, P. J.; Wang, D.; Frost, R. L.; J. Therm. Anal. Calorim. 2016, 126, 421.

34. He, H.; Duchet, J.; Galy, J. ; Gérard, J. F.; J. Colloid Interface Sci. 2006, 295, 202.

35. Li, Y. Q.; Ishida, H.; Chem. Mater. 2002, 14, 1398.

36. Yui, T.; Yoshida, H.; Tachibana, H.; Tryk, D. A.; Inoue, H.; Langmuir 2002, 18, 891.

Submitted: March 10, 2017 Published online: June 14, 2017 